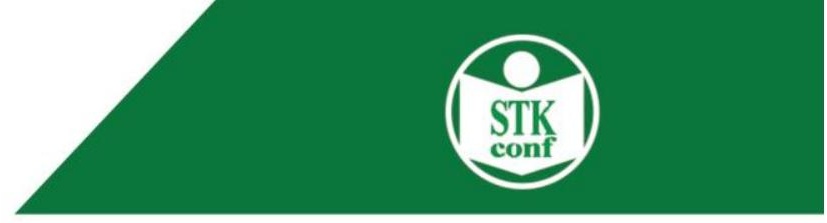

26-28 February, 2021

Amsterdam, Netherlands

\title{
Smart-in-Car Sensing
}

\author{
Mohammed Jabari ${ }^{1}$ and Musa Alrefaya ${ }^{2}$ \\ College of IT and Computer Engineering, Palestine Polytechnic University, Palestine
}

\begin{abstract}
.
The fact that most drivers these days carry their smartphones with them presents a great opportunity to develop a new type of applications that harvest and interpret the tremendous amount of data smartphones' sensors collect. Smart-in-car sensing presents smart solutions to several today's problems faced by drivers. One of the problems smartphones can help solve is traffic congestion. Fuel economy driving is another goal smartphone can help drivers achieve. Combining that with a solution to traffic congestion can contribute to low carbon dioxide emissions. A smooth and comfortable drive is something else smartphones sensors can help the drivers achieve. Moreover, smart-in-car sensing can prevent drowsy driving, which is a major cause of accidents. Furthermore, context-aware data provided by smart-in-car sensing is a gold mine of information that stakeholders can use to make better plans and to further the transition to smart cities. The proposed android application uses smartphones' accelerometer sensor to detect major events the car driver encounters during his daily drive. The application has to deal with many challenges, one of these is the fact that sensors' data, especially the accelerometer, produces too much noise, and to make use of the sensor's data there must be a way to filter out unwanted components of the data. Moreover, even low power sensors, like the accelerometer, in the fastest mode, can put considerable power demands on the smartphone's battery. Interpreting sensors' data and putting it into context is also a great challenge. The application was able to detect road bumps, driving behavior, and the effect of aggressive driving on the fuel consumption of the car. Moreover, the application can detect when a car enters a traffic jam, geotagging the location of the traffic jam, and share it with other drivers or simply upload it to the cloud.
\end{abstract}

Keywords: Smartphones, Sensors, Cars, Accelerometer, Mobile, Apps

\section{Introduction}

The widespread use of smartphones with their huge processing capabilities and the array of sensors they are shipped with, in addition to their mobility feature, have all opened the door for a multitude of applications in an unprecedented way. The fact that most drivers these days carry their smartphones with them, presents a great opportunity to develop a new type of applications that harvest and interpret the tremendous amount of data smartphones' sensors collect. Smart-in-car sensing uses smartphones' sensors to collect car movement data and puts it into context in a smart way that helps the driver. 


\section{$5^{\text {th }}$ International Conference On Applied Research In SCIENCE, TECHNOLOGY \& KNOWLEDGE}

accelerometer and gyroscope sensors are capable of detecting both acceleration and angular speed.

According to [10], aggressive driving can greatly affect the fuel economy of a car. Maintaining a constant speed on a journey, as opposed to frequent accelerating and decelerating, avoids fuel wastage. For example, hitting the brakes too often takes useful kinetic energy and turns it into waste heat. Cars' carbon dioxide emissions can be greatly lowered if drivers monitor their driving style. By using a smartphone's accelerometer and gyroscope sensors, it is possible to detect and keep track of a driver's driving patterns, which can be used to estimate the fuel savings he can make by changing his driving style, and thus ties the driver's carbon footprint to his wallet. The EPA estimates that aggressive driving can reduce gas mileage by up to $33 \%$ [10].

Insurance companies can also benefit from smart-in-car sensing by knowing more about a driver's behavior to assess the value of the insurance policy [3]. Smartin-car sensing can also detect when a driver falls asleep and can alert him to the danger. The American Automobile Association (AAA) estimates that one out of every six $(16.5 \%)$ deadly traffic accidents, and one out of eight $(12.5 \%)$ crashes requiring hospitalization of car drivers or passengers, is due to drowsy driving [11]. While smartin-car sensing can detect if the car is moving it can ask the driver to confirm he is awake on a regular basis using voice commands, and the driver must give a specific answer to a question in order to evaluate his mental concentration. Moreover, if the car is detected to be moving for a long time, the driver is instructed to stop the car and get some rest.

Smart-in-car sensing can also help drivers avoid bumpy roads. When a driver wants to avoid bumpy roads, like lots of speed bumps or cobbles or just plain old potholes, with all the data collected by the smartphone sensors, it is possible to determine if a road is bumpy or not and have an "avoid bumpy roads" option on the driving directions. Additionally, this kind of data can be sent to the city council or whatever party is responsible for the maintenance of the roads. Another way smart-incar sensing can be useful is in the case of accidents. Smartphone sensors can detect sudden and sharp movement changes, which is usually associated with car accidents. The smartphone can report the accident location to the authorities or even make a phone call for help.

\section{Related Work}

Recently, several interesting attempts to use smartphones' sensing capabilities to offer added-value service to the driver have emerged, e.g., [1][2][3][4][5][18][19]. In [1][2] authors mostly focus on car and smartphone interfacing, utilizing some builtin capabilities in the car, or in some cases, a specialized hardware. Although these approaches manage to offer some added-value service to the driver, the tight coupling with the car equipment restricts the use of such services to some car models only. In [3][4], authors discussed the potential of using smartphone sensors in building very useful Apps that aid the driver in their daily drive. Nevertheless, they did not provide a 


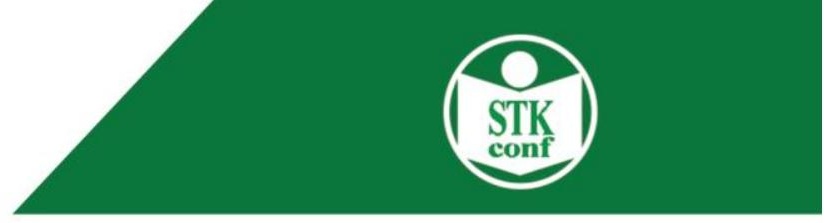

26-28 February, 2021

Amsterdam, Netherlands

is it possible to measure the accuracy of the data? Does looking for patterns help? If the driver is not interested in giving accurate or true data, what can be done?

Another concern is that smart-in-car sensing relies on the driver voluntarily giving his smartphone's sensor's data to the system. How can we incentivize the driver? Is the driver willing to sacrifice some of his privacy in exchange for some services? What kind of added-value services can really incentivize the driver to use the smart-incar sensing? Can a sense of social responsibility alone be enough?

Moreover, smart-in-car sensing, unlike most available mobiles Apps, which depends on drivers voluntarily entering data, will automatically collect sensors' data. The resulting huge quantity of data will need to be processed; some of the data processing can be done more efficiently on the cloud. This adds yet another challenge to this research, namely, building a cloud service capable of processing, analyzing, and interpreting the huge amount of sensors' data, and sharing it with community drivers and stakeholders.

As seen from the aforementioned challenges above, each one of them alone requires intensive research, thus, the main focus of this research will be on how to harvest sensors' data efficiently, isolate the noise, analyze and interpret to put in context, and share with other drivers and stockholders.

\section{Approach Overview}

This section contains a description of the approach used to detect significant driving events using smartphone's sensors. The focus of this research is to use low power sensors to detect car movements, mainly, the accelerometer. The accelerometer sensor is able to detect the acceleration forces applied to the three axes, namely, $(\mathrm{X}, \mathrm{Y}, \mathrm{Z})$. Fig (1) shows the sensor coordinate system. When the smartphone's orientation is portrait and the device is standing upright, axes run: $\mathrm{X}$ - right to left, $\mathrm{Y}-$ top to bottom, $\mathrm{Z}$ - Down to up, the coordinate system does not change when the device orientation changes [20]. In practice, the driver can put the device in any orientation he wishes, requiring extra work of the system to align the coordinate system with the car movement. For the simplicity of the research, it's assumed the device is aligned with the car's coordinate system as shown in fig (2). The car's coordinate system is defined by three orthogonal axes, $\mathrm{X}, \mathrm{Y}$, and $\mathrm{Z}$. X points to the right of the car, $\mathrm{Y}$ points toward the sky, and $\mathrm{Z}$ points toward traveling direction. 

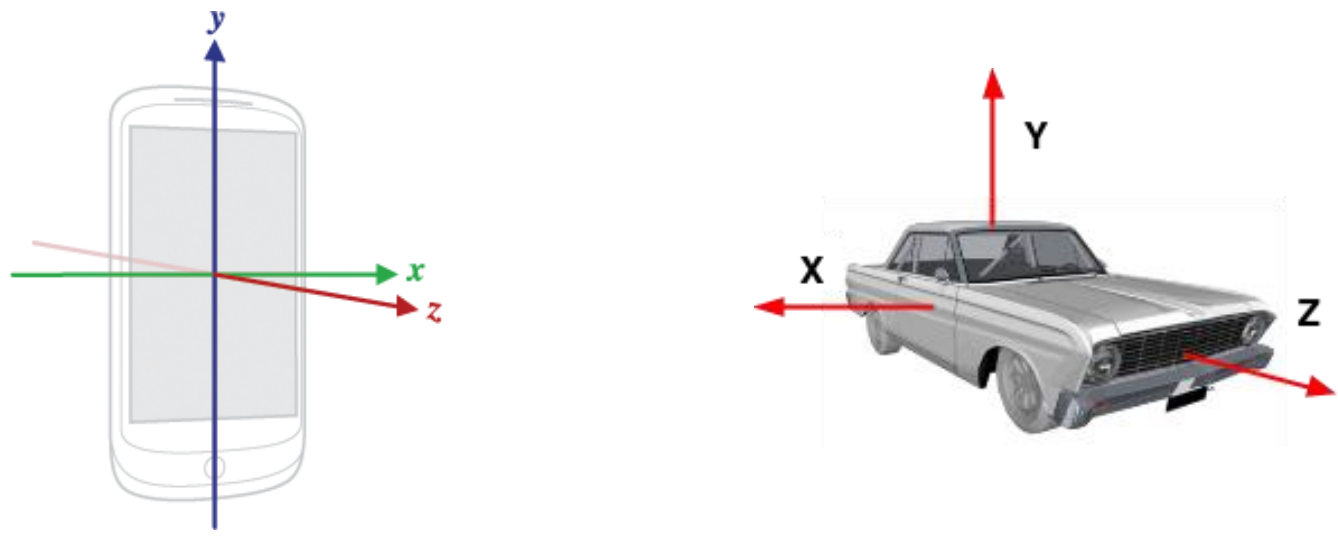

The smartphone will be mounted on the windscreen of the car using a special hanger as shown in fig (3), this arrangement, which requires little effort from the driver, ensures the smartphone's coordinate system is aligned with the car's coordinate system.

Fig (3) mounting of the smartphone

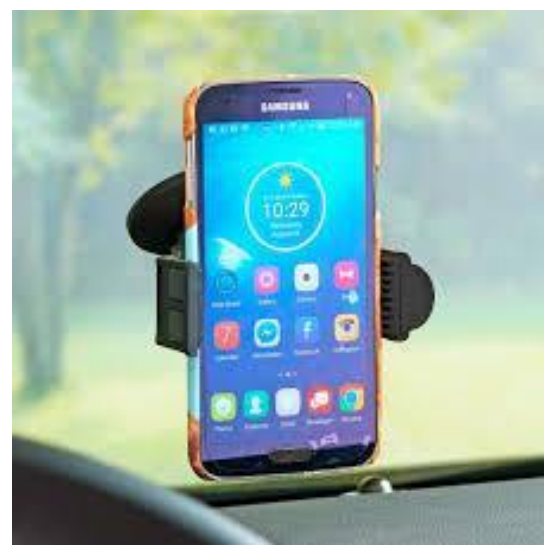

\section{Collecting Accelerometer Sensor Data}

The sampling rate of the accelerometer can affect energy consumption. In the fastest mode, low power sensors', i.e. accelerometer, energy consumption can reach $80 \%$ of that of a GPS sensor [4]. Hence a compromise must be made between increasing the harvesting of sensors' data in favor of accuracy and making do with a low sampling rate in favor of low power consumption. After doing several experiments, android's SENSOR_DELAY_GAME mode was used; in this mode, the accelerometer's sampling rate was about 50 samples per second [20], which offers accepted accuracy with suitable power demands. An Android app was developed to record and log accelerometer sensor data. The smartphone used was Samsung Galaxy S4 running Android 5.2. The experiment was done on a sedan car, three journeys on the same route were done, and each journey took about 5 minutes. Part of the sample-collected data is 
shown in fig (4). It's worth noting from fig (4) that the reading of the accelerometer is fluctuating very rapidly, it's due to the fact that the accelerometer is very sensitive to small movements, even if the car is not moving at all, the accelerometer still shows some variations in the readings. To overcome this problem and to only account for car major movements changes, which is the aim of this research, a low-pass filter is applied to filter out noisy unwanted components of the data. A low-pass filter is usually used when we want to filter out high-frequency readings and only account for more persistent changes, in which case, the major changes in the acceleration of the car [21].

Fig (4) Accelerometer's sensor data for the car journey (not filtered)

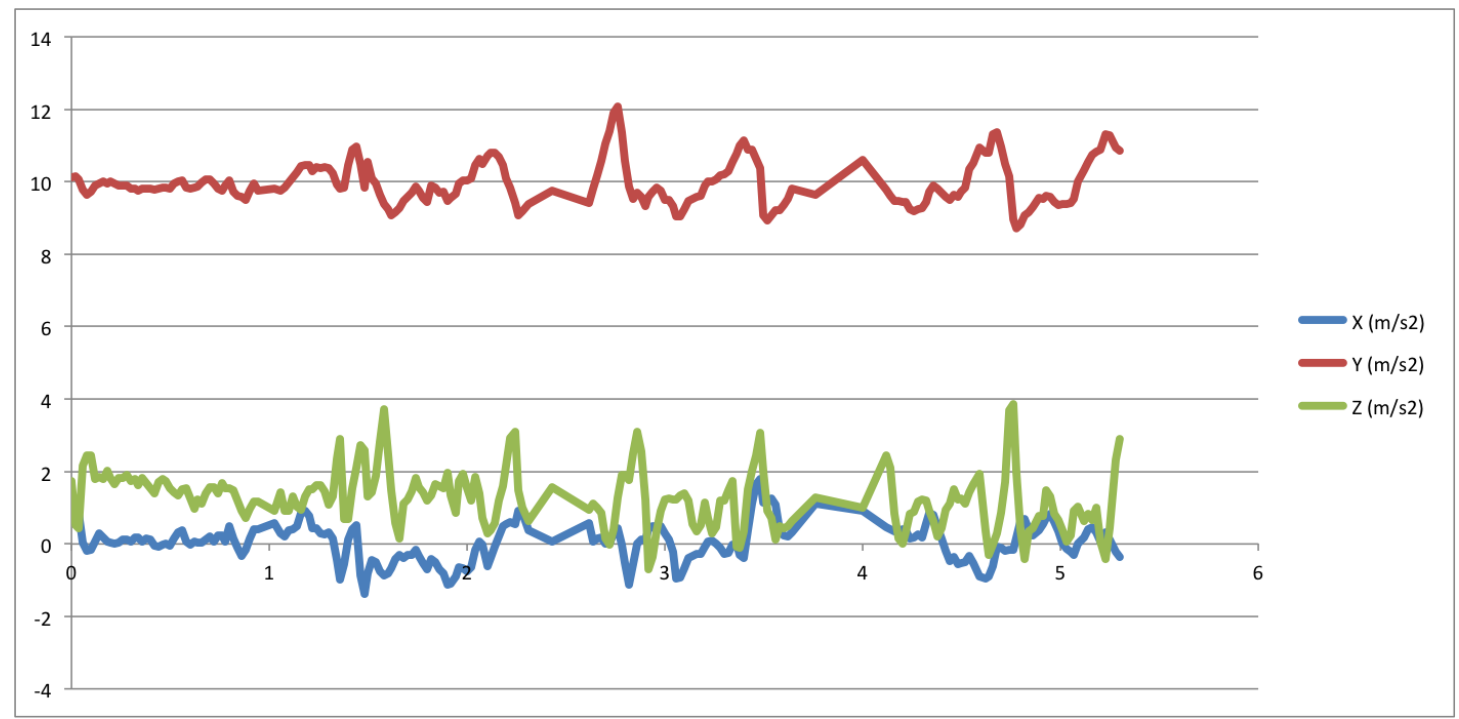

The moving average is used as a simple and efficient low-pass filter [21], the moving average can be applied for $n$ values of the accelerometer, for example: $Y 0, Y 1$, $\mathrm{Y} 2 \ldots \mathrm{Yn}_{-1}$, where $\mathrm{Y}$ is the acceleration in the $\mathrm{Y}$ direction (Sky). For these values the average formula will be:

$$
\operatorname{avg}=\frac{1}{n} \sum_{i=0}^{n-1} \quad Y i
$$

To account for the new coming values and to calculate the moving average, a new value is added to the sum and the old value dropped out. So, the equation becomes:

$$
M a v g=M a v g, p r e v+\frac{Y n}{n}-\frac{Y i-1}{n},
$$

Where Yn is the new value and Yi-1 is the old value. Fig (5) shows the sample data after applying the moving average. 
Fig (5) Accelerometer's sensor data for the car journey (filtered)

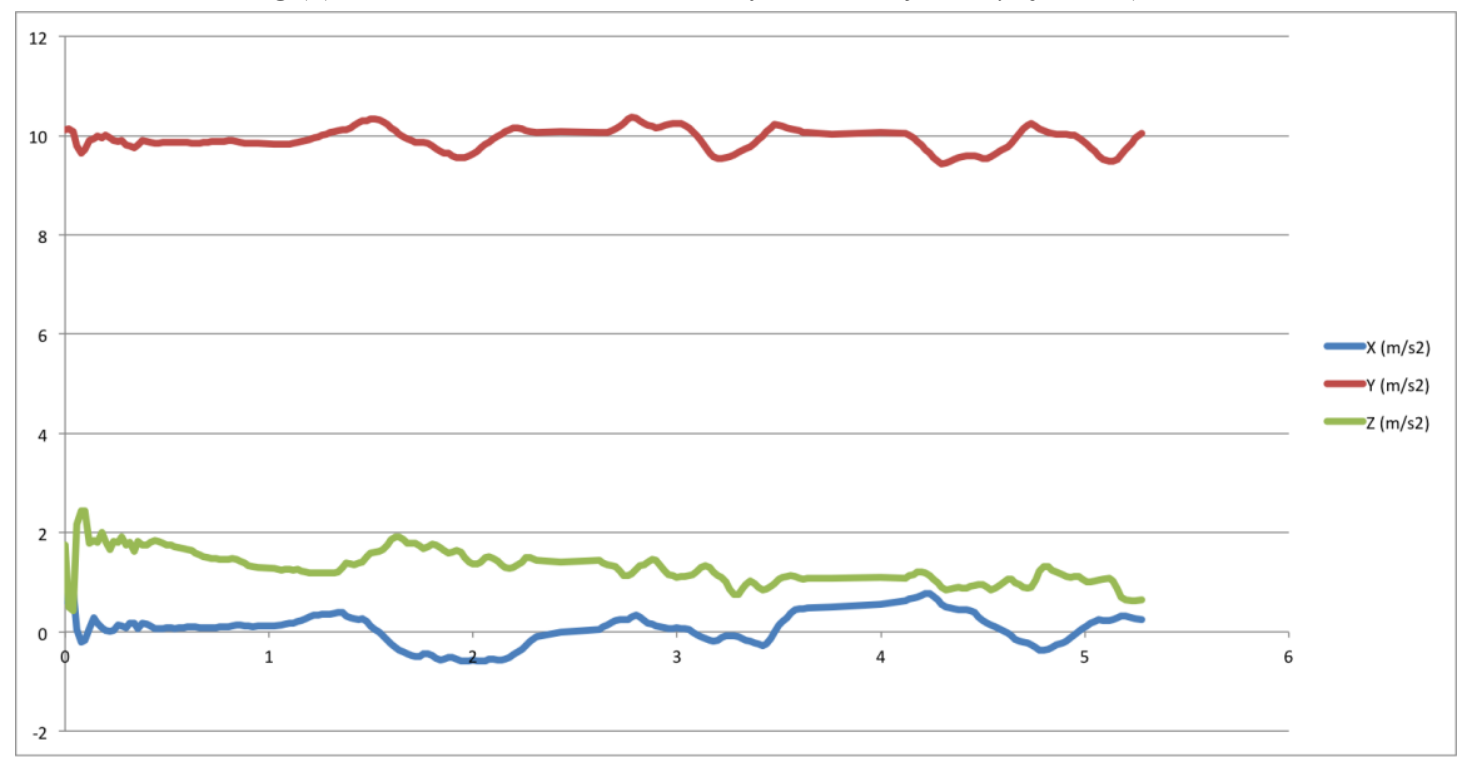

\section{Interpreting the Data}

Raw sensors' data, especially the accelerometer and gyroscope, contains too much noise and needs filtering and processing before it can be used to extract any useful information. Furthermore, inferring the context using sensors' data has many complications and offers a big challenge. Several methods were employed in order to distinguish and detect different events the car went through using sensor's data. It's worth noting that the experiments were performed with the intention to detect specific events, and no time or effort was enough to account for all kind of different events.

\subsection{Detecting Road Condition:}

Detecting the road conditions, which includes road bumps and holes, requires special attention to the accelerometer readings in the Y direction (Sky) as shown in fig (2). The kind of forces that will be exerted on the car in such cases will be more obvious in the Y direction, fig (6) shows the change in the acceleration value for $\mathrm{Y}$ in case the car just passed a road bump. The change in the values is clear, which resembles two peaks sinusoidal signal, the first peak is formed when the car's front wheels pass the bump, the second, when the car's rear wheels pass. Detecting such a signal in real time on a smart device by passing the data to a sinusoidal approximation equation can be used. However, considering the fact that the accelerometer sample rate is about 50 samples per second, performing such calculation on a smartphone for every value coming from the accelerometer requires intensive processing which will cause the whole application to run slow. 


\section{$5^{\text {th }}$ International Conference On Applied Research In SCIENCE, TECHNOLOGY \& KNOWLEDGE}

Fig (6) The change of acceleration in $Y$ direction in case of road bump

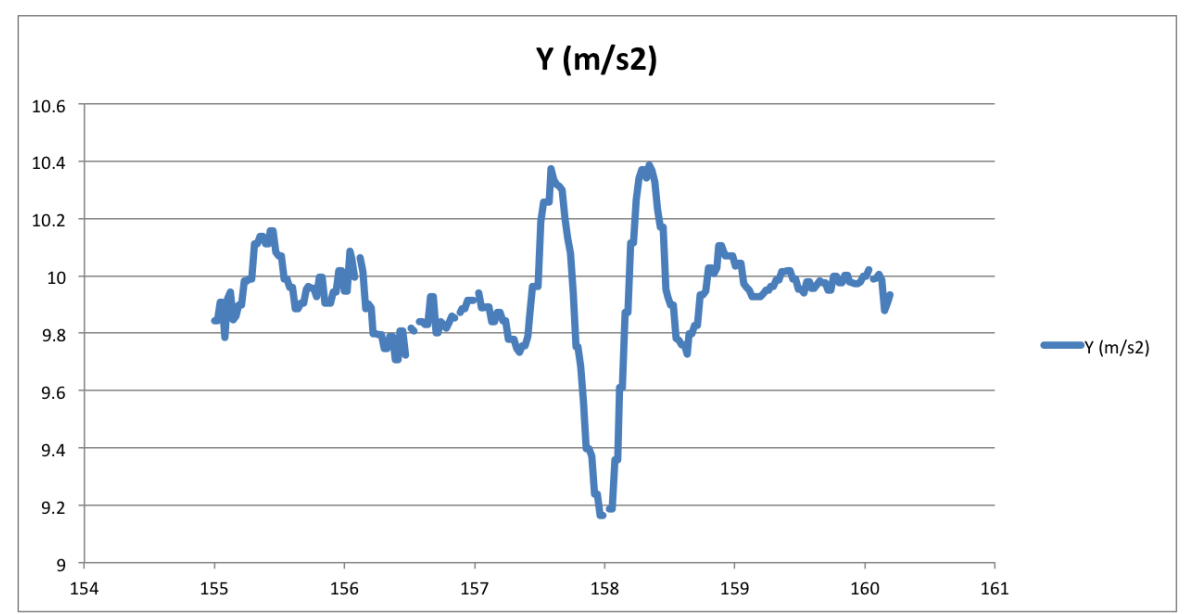

Another simpler and more efficient approach is to look for two successive peak values in a specific time period. After performing several experiments on different kinds of road bumps with different passing speeds, it was found that the time period between the two peaks was neither less than 500 milliseconds nor greater than 2000 milliseconds, and the peak value was more than $10.2 \mathrm{~m} / \mathrm{s}^{2}$. The following shows part of the algorithm used to detect a road bump.

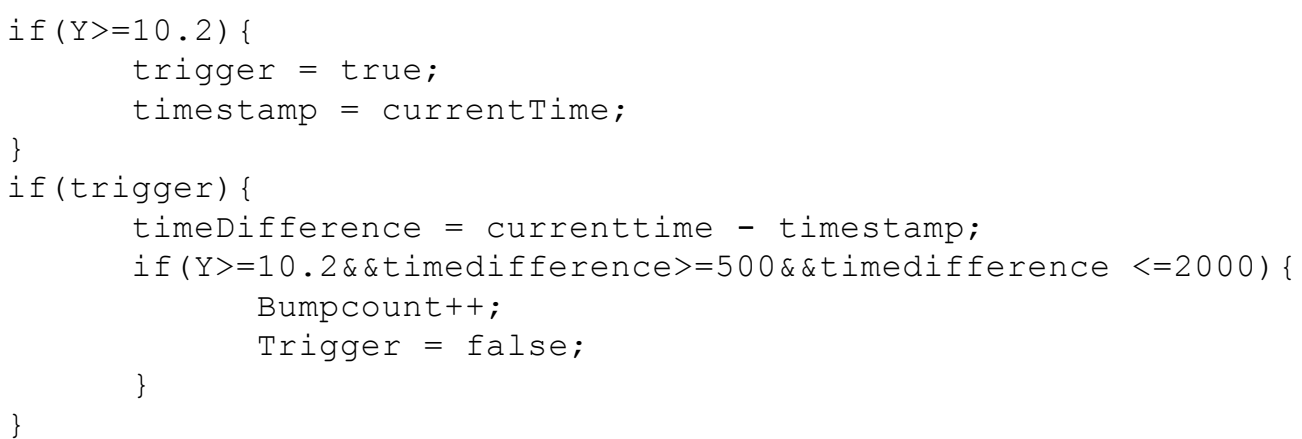

In the above algorithm, $\mathrm{Y}$ is the acceleration value coming from the accelerometer sensor; timestamp is the time the first peak value appears. Applying this algorithm to the data collected from the three journeys the application was able to detect $5 \mathrm{road}$ bumps each time.

\subsection{Detecting Driving Behavior}

Driving behavior can be categorized as normal or aggressive, aggressive driving is characterized by frequent acceleration and deceleration in a short period of time, the accelerometer sensor can detect the acceleration and deceleration of the car in the driving direction. Fig (7) shows the accelerometer sensor data for the acceleration of the car in the driving direction $(\mathrm{Z})$. For this data, the car driver, on purpose, tried to be 


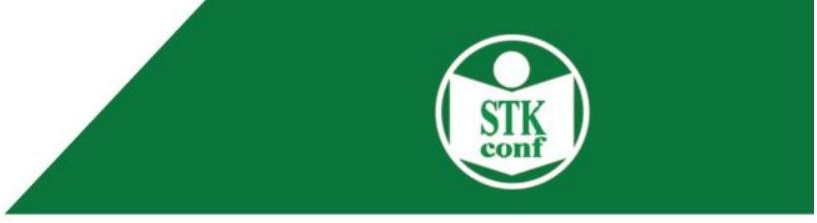

26-28 February, 2021

Amsterdam, Netherlands

aggressive in driving, as shown from the figure, there are sharp changes in the accelerometer data, specifically, when the car was decelerating and accelerating. When the car moves at almost a steady speed, no significant changes to $Z$ values can be detected. It's only when the car is accelerating or decelerating. The data collected from the experiment shows that aggressive driving is considered aggressive if a sharp change in acceleration is detected in less than a second ( 1 second). As shown in fig (7) there is a significant change in the acceleration in the $\mathrm{Z}$ direction in less than a second. In order to be able to detect these changes, every value from the previous second is compared to the new arriving value coming from the sensor. If the difference between the two values is bigger than a specific threshold it's considered an event of aggressive driving as shown from the following equation

$$
\text { AccDiff }=Z_{n}-Z_{n-k}
$$

Where AccDiff is the difference of acceleration between $\mathrm{Zn}$ and $\mathrm{Zn}-\mathrm{k}$, where $\mathrm{Zn}$ is the acceleration at time $\mathrm{n}$ and $\mathrm{Zn}-\mathrm{k}$ is the acceleration at time $\mathrm{n}-\mathrm{k} . \mathrm{K}=1$.

Fig (7) Accelerometer' data for aggressive driving

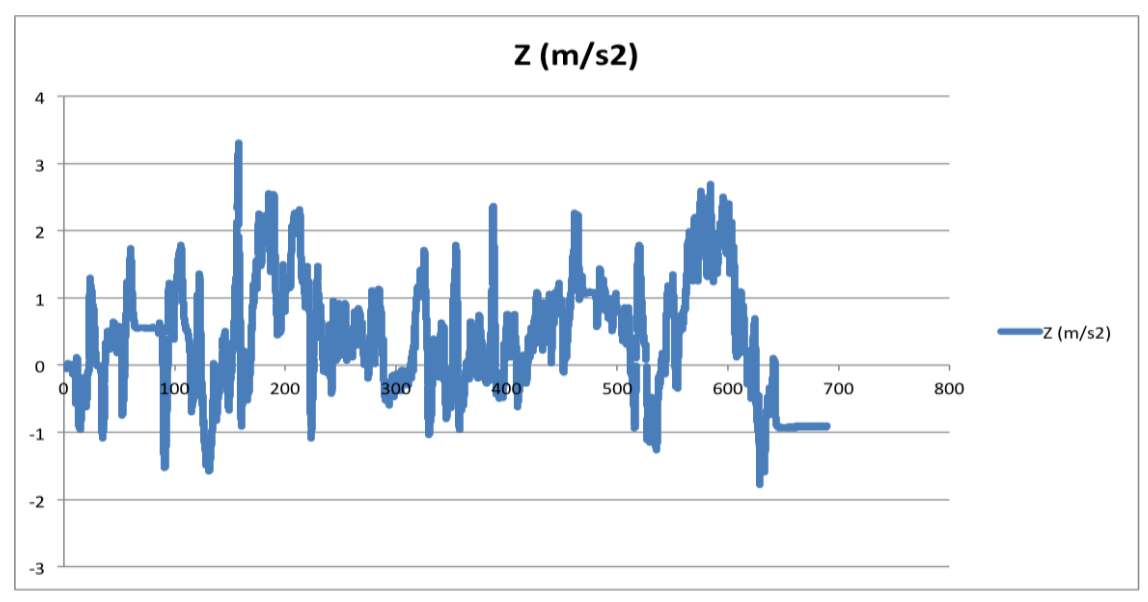

\subsection{Driving Behavior and Fuel Economy}

According to [10], driving behavior can significantly affect fuel economy of the car. Frequent acceleration and deceleration of the car can take useful kinetic energy and convert it into heat. In order to evaluate the effect of aggressive driving on the fuel economy of the car, two journeys on a $3 \mathrm{~km}$ route were done. To avoid traffic jams and for safety reasons, the two journeys were done after midnight. For both journeys, the car's fuel tank was pumped out and its volume was recorded and then the fuel was put back to the car's tank, the same was done after each journey. The first journey was smooth and normal driven while the second journey the car was driven in an aggressive 


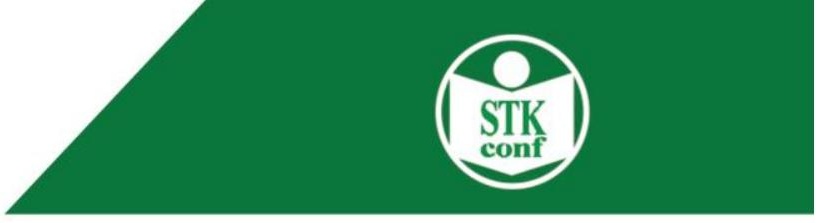

26-28 February, 2021

Amsterdam, Netherlands

way. Table (1) shows the results of the experiment, fig (8) shows the accelerometer data for the first, smooth journey, fig (9) shows the accelerometer data for the second, aggressive journey.

Table (1) Fuel consumption for the smooth and aggressive journeys

\begin{tabular}{|l|l|l|l|}
\hline Journey & Fuel in tank before & Fuel in tank after & Fuel Consumed \\
\hline Smooth & 9.4 Liters & 9.0 Liters & 0.4 Liters \\
\hline Aggressive & 6.3 Liters & 5.6 Liters & 0.7 Liters \\
\hline
\end{tabular}

As shown from table (1), it's clear and evident the effect, aggressive driving can impose on the consumption of fuel, for only $3 \mathrm{~km}$ journey, the driver can save up to 0.3 liters of fuel if he drives in a smooth way. The Application enables the driver to monitor his driving habits and as a result, act when aggressive driving behavior is detected if he wants to save fuel and lower his carbon dioxide footprint.
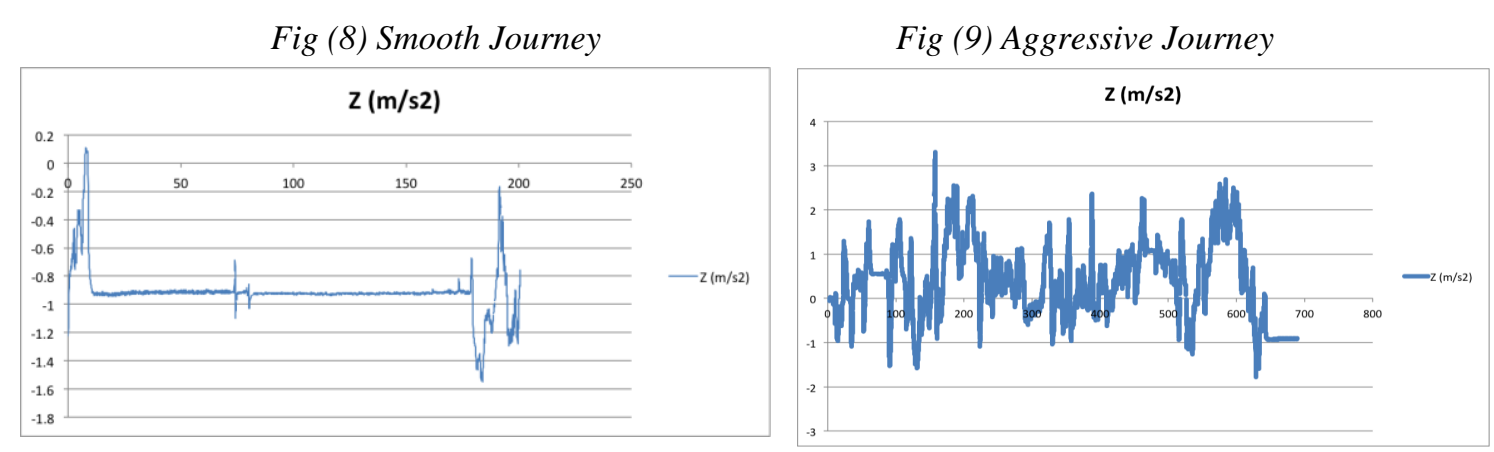

\subsection{Detecting Traffic Jams}

Whenever a car enters a traffic jam, it's either, at a full stop, or moving very slowly. The car will alternate between these two states until it exits the traffic jam. If the car encountered a full stop more than $n$ times in a short period of time $t$, then we can assume the car just entered a traffic jam. Smart-in-car sensing, using the accelerometer, can detect the change of states between moving and stopping, by counting the number of stops within a period of time, if it matches a certain threshold then the system can safely assume it's a traffic jam. GPS in the smartphone can be used to geotag the location as a traffic jam location. Unlike most smartphone's applications today, which relies on the driver manually reporting traffic jams to some system, smartin-car sensing can automatically share this information with other drivers driving on the same road or even, send it to a cloud service where it can be used by whatever party is responsible for the planning of the roads. 


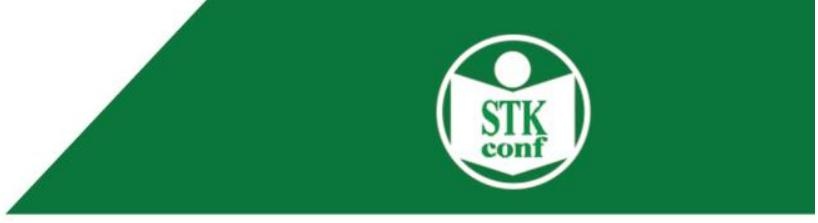

26-28 February, 2021

Amsterdam, Netherlands

Figure (10) shows the acceleration value for $Z$ (the driving direction), it's obvious the alternate change in its value when the car changes state from moving slowly to full stop.

Fig (10) Accelerometer data for frequent car stops

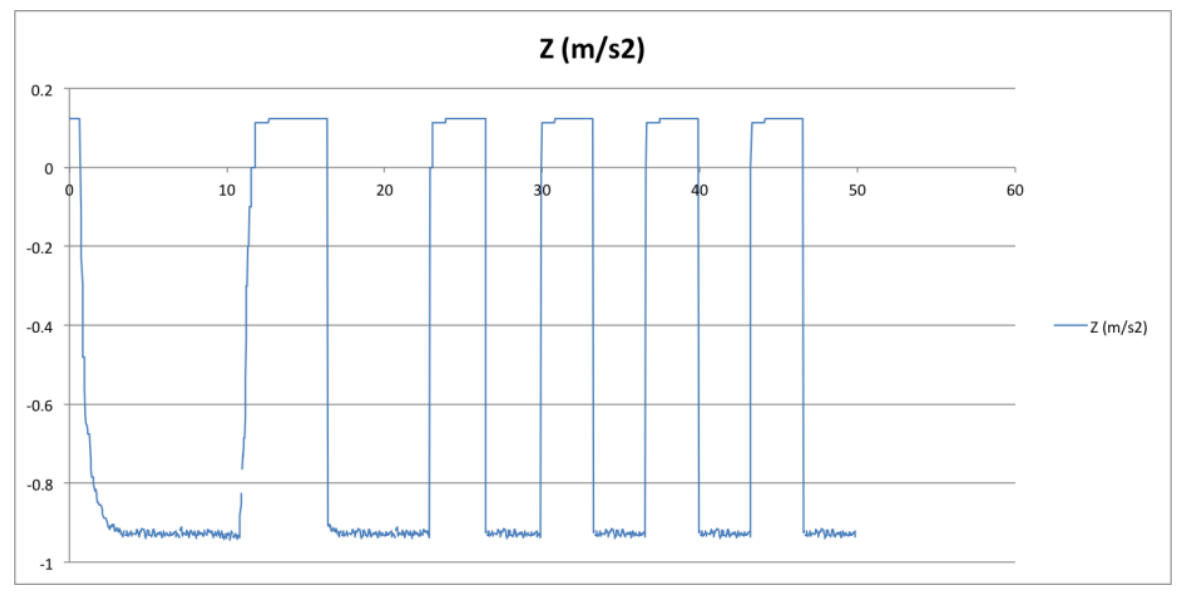

\section{Conclusion and Future Work}

Smartphones today are equipped with an array of sensors, specifically an accelerometer and gyroscope; these sensors can be used to detect the changes in movement of a car. The fact that most people carry with them their smartphones when driving opens the door for the possibility for the use of these smartphones' sensors to detect and record major driving events. Road conditions, aggressive driving and fuel economy in addition to traffic jams, all can be detected and recorded using today's smartphones' sensors.

The proposed application, Smart-in-Car sensing, was able to record and interpret different events the driver encountered during his daily drive. Smart-in-car sensing was able to detect road bumps, driving behavior, and the effect of aggressive driving on the fuel consumption of the car. Moreover, smart-in-car sensing can detect when a car enters a traffic jam, geotagging the location of the traffic jam, and share it with other drivers or simply upload it to the cloud.

Still, many questions regarding the use of smartphones' sensors in aiding the driver in his daily drive need to be answered. Drivers who are willing to give their sensors' raw data to the system need to make sure their privacy is preserved, e.g., their location information can't be shared without their consent. In some cases, restricted context information can be inferred from other available context information. How can we anonymize the collected data? What kind of information can be used to infer a user's location, habits, or identity? For what extent the user is willing to trade privacy for application functionality?

Sensed data integrity is another important issue; any application based on sensors' data will be useless if the collected sensors data can't be trusted in terms of accuracy and timeliness. Unlike wireless sensors' networks, where the system consists of 


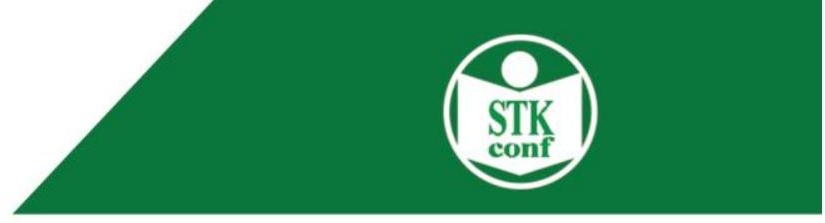

26-28 February, 2021

Amsterdam, Netherlands

stationary sensors distributed in an environment, smart-in-car sensing relies totally on the driver's smartphone sensors. In which case the owner of the smartphone - the driver - can manipulate and interfere with sensors' data. How is it possible to measure the accuracy of the data? Does looking for patterns help? If the driver is not interested in giving accurate or true data, what can be done?

Furthermore, the proposed application requires a special arrangement for the smartphone in the car to be aligned with the car's coordinate system. This arrangement, which requires little effort from the driver, can be annoying to the driver, and sometimes the driver may forget to do it. Yet, this adds another challenge, i.e. how to align the smartphone's coordinate system with the car's coordinate system without any effort or intervention from the driver?

Moreover, smart-in-car sensing, unlike most available mobiles Apps, which depends on drivers voluntarily entering data, will automatically collect sensors' data. The resulting huge quantity of data will need to be processed; some of the data processing can be done more efficiently on the cloud. This adds yet another challenge to this research, namely, building a cloud service capable of processing, analyzing, and interpreting the huge amount of sensors' data, and sharing it with community drivers and stakeholders.

\section{REFERENCES}

[1] Jorge Zaldivar, Carlos T. Calafate, Juan Carlos Cano, Pietro Manzoni (2011). "Providing Accident Detection in Vehicular Networks Through OBD-II Devices and Android-based Smartphones", IEEE 36th Conference on Local Computer Networks

[2] C. Campolo, A. lera, A. Molinaro, S. Y. Paratore and G. Ruggeri. (2012). "SMaRTCaR: An integrated smartphone-based platform to support traffic management applications," 2012 First International Workshop on Vehicular Traffic Management for Smart Cities (VTM), Dublin, pp. 1-6.

[3] Sarfraz Nawaz, Christos Efstratiou, and Cecilia Mascolo. (2016). "Smart Sensing for the daily drive" In IEEE Pervasive Computing Magazine. January-March 2016 Issue.

[4] Sarfraz Nawaz, Cecilia Mascolo. (2014). "Mining User's Significant Driving Routes With Low-Power Sensors" Proceedings of the ACM International Conference on Embedded Networked Sensor Systems (SenSys 2014). Memphis, Tennessee, USA. November 2014.

[5] Chuang-Wen You, Martha Montes-de-Oca, Thomas J. Bao, Nicholas D. Lane, Hong Lu, Giuseppe Cardone, Lorenzo Torresani, and Andrew T. Campbell. (2012). CarSafe: a driver safety app that detects dangerous driving behavior using dualcameras on smartphones. In Proceedings of the 2012 ACM Conference on Ubiquitous Computing (UbiComp '12). ACM, New York, NY, USA, pp. 671-672.

[6] Khairul Muzzammil Saipullah, Ammar Anuar, Nurul Atiqah Ismail and Yewguan Soo. (2012). "MEASURING POWER CONSUMPTION FOR IMAGE PROCESSING ON ANDROID SMARTPHONE", American Journal of Applied Sciences, 9(12), 2052-2057 


\section{$5^{\text {th }}$ International Conference On Applied Research In SCIENCE, TECHNOLOGY \& KNOWLEDGE}

[7] INRIX Driving Intelligence "Key Findings: INRIX 2015 Traffic Scorecard" http://inrix.com/scorecard/key-findings-us/, accessed: 2/12/2016

[8] US Department of Energy, http://energy.gov/eere/vehicles/fact-897-november-2-2015fuel-wasted-traffic-congestion, accessed: 2/12/2016

[9] The Wall Street Journal, "One Driver can Stop the Traffic Jam" http://www.wsj.com/articles/one-driver-can-prevent-a-traffic-jam-1476204858, accessed: 2/12/2016

[10] Energy Efficiency and Renewable Energy, US Department of Energy, "Driving more Efficiently", http://www.fueleconomy.gov/feg/driveHabits.jsp, accessed: 2/12/2016

[11] American Automobile Association Foundation for Traffic Safety, 2010. "Asleep at the wheel: the prevalence and impact of drowsy driving," http://www.aaafoundation.org/pdf/2010DrowsyDrivingReport.pdf, accessed 2/12/2016

[12] The solution for traffic congestion, http://kottke.org/16/09/phantom-intersectionstraffic-snakes-and-the-simple-solution-to-traffic, accessed: 2/12/2016

[13] Fake Gps Location, https://play.google.com/store/apps/details?id=com.kuci.gpspicker\&hl=en

[14] Fake GPS Tilt to Move, https://play.google.com/store/apps/details?id=com.neptuns.fakegpstilttomove\&hl=en

[15] Alastair R. Beresford, Andrew Rice, Nicholas Skehin, and Ripduman Sohan. (2011). MockDroid: trading privacy for application functionality on smartphones. In Proceedings of the 12th Workshop on Mobile Computing Systems and Applications (HotMobile '11). ACM, New York, NY, USA, 49-54.

DOI=http://dx.doi.org/10.1145/2184489.2184500

[16] Apu Kapadia, David Kotz, Nikos Triandopoulos. (2009) "Opportunistic sensing: Security challenges for the new paradigm", Communication Systems and Networks and Workshops, COMSNETS 2009. First International

[17] Zhi Xu and Sencun Zhu. (2015). SemaDroid: A Privacy-Aware Sensor Management Framework for Smartphones. In Proceedings of the 5th ACM Conference on Data and Application Security and Privacy (CODASPY '15). ACM, New York, NY, USA, 61-72. DOI=http://dx.doi.org/10.1145/2699026.2699114

[18] Avik Ghose et.al. (2012). Road condition monitoring and alert application: Using invehicle Smartphone as Internet-connected sensor. Pervasive Computing and Communications Workshops (PERCOM Workshops), IEEE International Conference.

[19] Derick A. Johnson, Mohan M. Trivedi. (2011). Driving style recognition using a smartphone as a sensor platform. Intelligent Transportation Systems (ITSC), 14th International IEEE Conference on 


\section{$5^{\text {th }}$ International Conference On Applied Research In SCIENCE, TECHNOLOGY \& KNOWLEDGE}

[20] Sensor Event, official android developer guide website, https://developer.android.com/reference/android/hardware/SensorEvent.html, accessed 2/12/2016

[21] Digital Modulations using Matlab: Build Simulation Models from Scratch, page: 78, Mathuranathan Viswanathan, ISBN-10: 152149388X 